\title{
A three-dimensional head-mounted display system (RoboSurgeon system) for gasless laparoendoscopic single-port partial cystectomy
}

\author{
Yasuhisa Fujii, Kazunori Kihara, Soichiro Yoshida, Junichiro Ishioka, Yoh Matsuoka, Noboru Numao, Kazutaka Saito \\ Department of Urology, Tokyo Medical and Dental University Graduate School, Tokyo, Japan
}

Videosurgery Miniinv 2014; 9 (4): 638-643

DOI: $10.5114 /$ wiitm.2014.44407

\begin{abstract}
We developed a new three-dimensional (3D) head-mounted display (HMD) system (RoboSurgeon system) that combines a high-definition 3D organic electroluminescent HMD with a high-definition 3D endoscope and applies it to minimally invasive surgery. This system presents the surgeon with a higher quality of magnified 3D imagery in front of the eyes, regardless of head position. We report 5 cases of RoboSurgeon gasless laparoendoscopic single-port partial cystectomy, which is carried out as part of our selective bladder-sparing protocol, with a technique utilizing both an intravesical and extravesical approach. While carrying out the surgery, the system provides the surgeon with both excellent 3D imagery of the operative field and clear imagery of the cystoscopy. All procedures were safely completed and there were no complications except for a case of postoperative lymphorrhea. Our experience shows that the 3D $H M D$ system might facilitate maneuverability and safety in various minimally invasive procedures.
\end{abstract}

Key words: head-mounted display, minimally invasive surgery, three-dimensional high-definition endoscope, cystoscope, bladder cancer, partial cystectomy.

\section{Introduction}

Radical cystectomy (RC) plus urinary diversion has long been the reference standard treatment for muscle-invasive bladder cancer (MIBC). However, $\mathrm{RC}$ is associated with high complication rates and can compromise quality of life as a result of longterm effects on urinary, gastrointestinal, and sexual function as well as changes in body image. Recently, various modalities of bladder-sparing approaches have been investigated and their benefits appreciated. Many of these protocols consist of transurethral resection of bladder tumor (TURBT) plus full-dose chemoradiotherapy (CRT) [1]. Their limitations are 1) MIBC recurrence in the preserved bladder mainly due to subclinical residual disease in the original
MIBC site and 2) potential lack of curative intervention to regional lymph nodes [2, 3]. Since 1997, we have carried out a selective bladder-sparing protocol incorporating consolidative partial cystectomy (PC) with pelvic lymph node dissection (PLND) after debulking TURBT and low-dose chemoradiotherapy (LCRT) [2-4]. Only patients who desire bladder preservation and who meet all of the PC criteria selectively undergo PC with PLND with curative intent [24]. The PC criteria include: 1 ) intravesically unifocal tumors ( $<25 \%$ of the bladder in area, excluding the bladder neck and trigone); 2) no involvement of the bladder neck or trigone; and 3) no residual tumor, or only small amounts of residual non-muscle-invasive bladder cancer (NMIBC) in the original MIBC site at restaging TURBT after LCRT. The PC with PLND could

\section{Address for correspondence}

Kazunori Kihara MD, PhD, Department of Urology, Tokyo Medical and Dental University, 1-5-45 Yushima, Bunkyo-Ku, Tokyo 113-8519,

Japan, phone: +81 35803 5295, fax: +8135803 5295, e-mail: k-kihara.uro@tmd.ac.jp 
contribute to eradication of subclinical residual disease in original MIBC sites and micrometastases in pelvic lymph nodes and has been carried out using techniques of gasless single-port surgery, a minimally invasive surgery via a single port without $\mathrm{CO}_{2}$ gas insufflation $[2,5,6]$.

In 2011, we developed an affordable new threedimensional (3D) vision system (RoboSurgeon system) with technical support from Sony Corporation, which is a combination of a high-definition 3D organic electroluminescent head-mounted display (HMD) (HMZ-T1; Sony Corporation, Tokyo, Japan) and a high-definition 3D endoscope (Shinko Optical, Tokyo, Japan, and Endoeye flex 3D deflectable videoscope, Olympus, Tokyo, Japan) and first applied this system to gasless single-port radical nephrectomy [7]. Since 2013, the 3D HMD for medical use (HMM-3000MT; Sony Corporation, Tokyo, Japan) has been commercially available and we have adopted it. This system provides surgeons with high-quality $3 \mathrm{D}$ imagery right before their eyes, regardless of head position, and direct vision is readily available by glancing downward. Wearing the HMD, the lead surgeon and the assistants can simultaneously and continuously monitor the same image displayed by the HMD. Furthermore, this system is equipped with a 'Picture in Picture' feature, which enables a second image (such as from cystoscopy) to appear as a window while the image from the laparoscopy is kept as the main image. Each participant can independently rearrange the images comprising the composite image displayed on his HMD depending on the surgical step. The excellence of the HMD as a personal imaging monitoring system led us to apply this system to other gasless single-port procedures. Here, we present 5 cases of patients with MIBC undergoing RoboSurgeon gasless single-port partial cystectomy with a technique utilizing both an intravesical and extravesical approach.

\section{Case reports}

\section{Surgical technique}

Informed consent was obtained after a complete description of the various surgical procedures, including the gasless single-port surgery using the RoboSurgeon system, which was carried out with the approval of our university's ethics committee. All procedures were carried out by a surgeon who was experienced in gasless single-port surgery. The techniques of gasless single-port surgery have been presented previously $[5,6]$.

An outline of the surgery is shown in Figure 1. While under general anesthesia, the patient is placed in the lithotomy position. All surgeons wear an $\mathrm{HMD}^{* *} 1^{\star *}$ (Photo 1). A lower abdominal midline incision of around $4 \mathrm{~cm}$ is made and a single port is prepared by placement of an Alexis wound retractor (Applied Medical, Irvine, CA, USA). All devices, including a high-definition $3 D$ endoscope, are inserted through the single port. All surgeons then begin to view the 3D imagery via the HMD. Whenever the surgeon receives the devices and inserts them through the port, the angle of sight is moved downward to obtain direct vision. Immediately after device insertion, every maneuver is carried out using a clear 3D image of the device which appears on the display. The 'Picture in Picture' feature is used to simultaneously show both images of the laparoendoscope and cystoscope (Photo 2).

Partial cystectomy is typically performed using an extraperitoneal approach. After dissection of the space of Retzius and exposure of the anterior surface of the bladder, the Iglesias resectoscope is placed into the urethra and cystopanendoscopy is performed. On cystoscopy, their original MIBC site typically appears like a scar because these patients must meet the $P C$ criteria that there is no residual tumor or only small amounts of residual NMIBC in the original MIBC site after LCRT. Then, under laparoendoscopy, the bladder is freed necessarily and sufficiently beyond the site of the tumor, which can easily be recognized from outside of the bladder by the transmitted light of the cystoscope passing through the bladder wall (Photo 2). For tumors located at the dome, the peritoneum is opened. The fat and the peritoneum over the site of the tumor is left attached to the bladder. Under cystoscopy, the proposed excision line around the tumor scar with a margin of approximately $1 \mathrm{~cm}$ is scored on the bladder surface using a Collins knife electrode. Next, the Collins incision is deepened along the proposed resection margin through the serosa until full thickness is achieved (Photo 2). The TUR surgeon carries out this procedure while viewing the cystoscopic image as the main image and the laparoendoscopic image as the second image of the 'Picture in Picture' on the HMD. Other surgeons see the laparoendoscopic image as the main image and the cystoscopic image as the second image to confirm that there is 


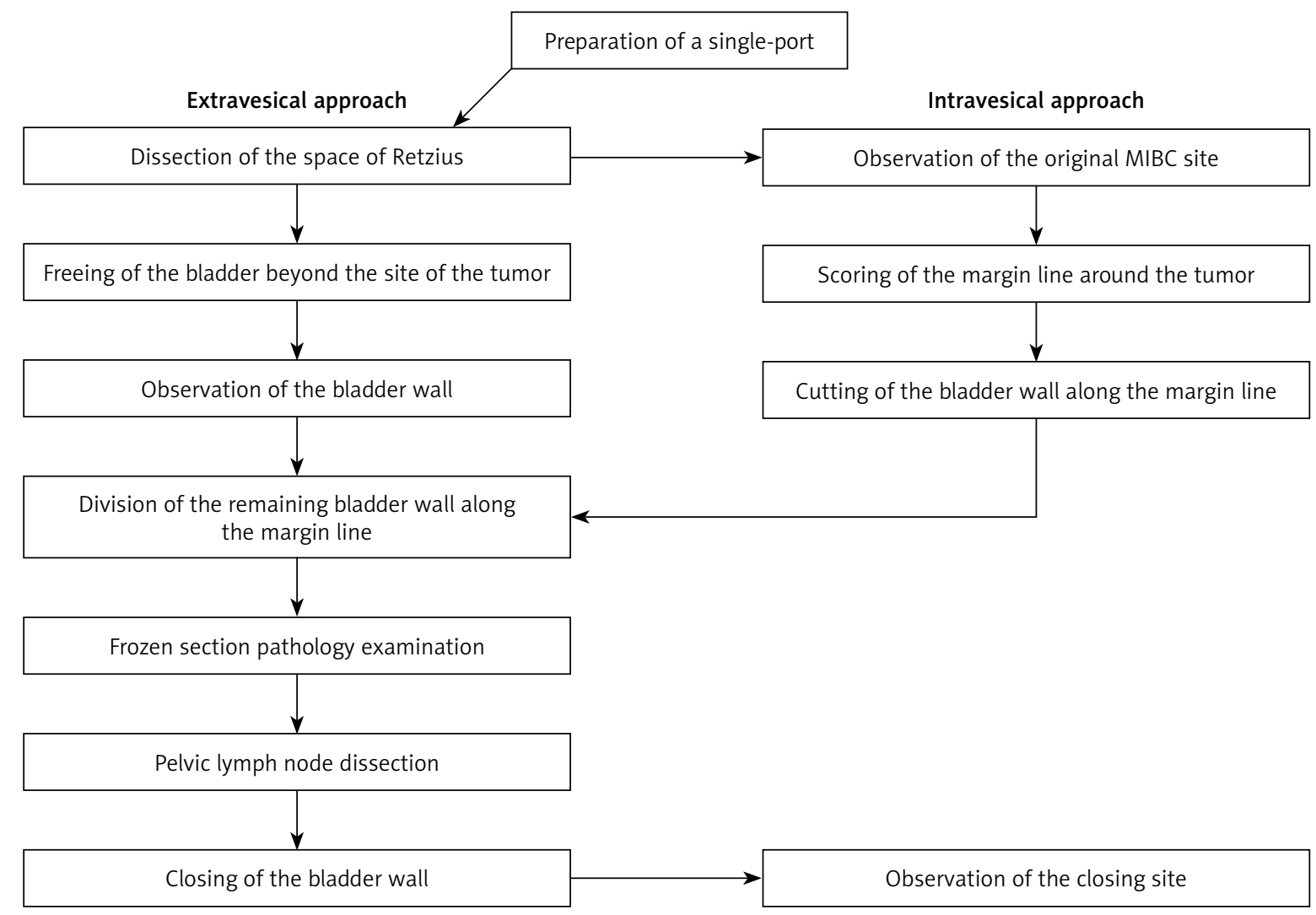

Figure 1. Outline of RoboSurgeon gasless single-port partial cystectomy
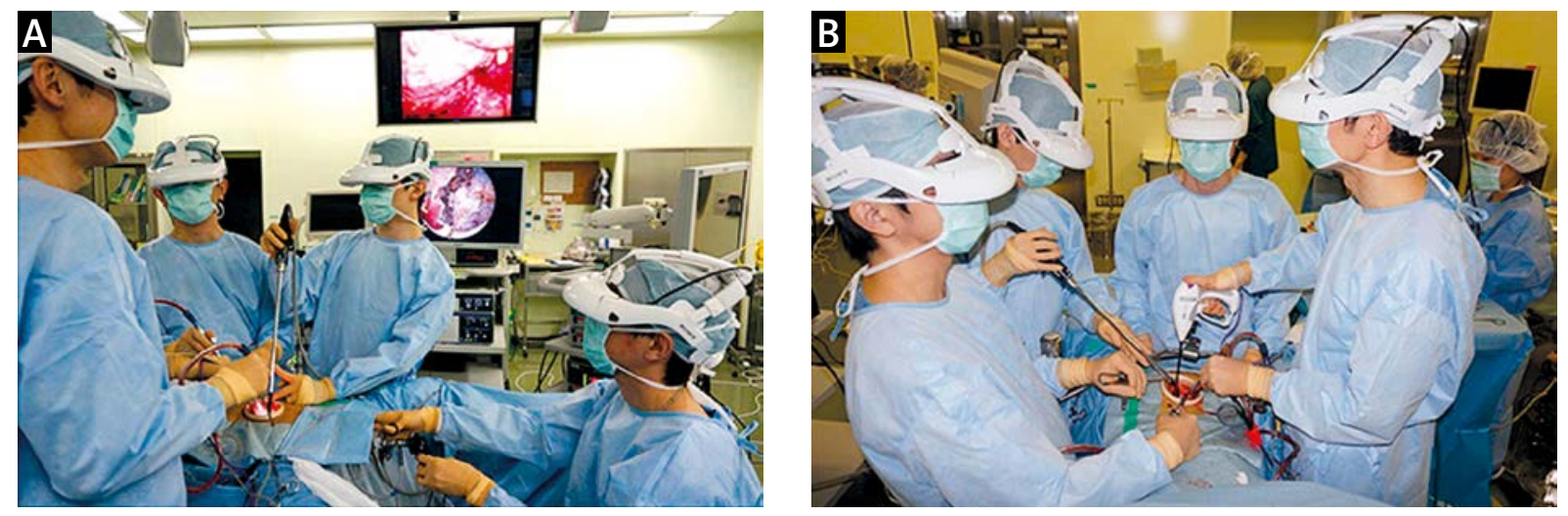

Photo 1. Photograph of RoboSurgeon gasless laparoendoscopic single-port partial cystectomy. All surgeons, including the TUR surgeon, wear a head-mounted display (A). After the intravesical approach is finished, the remaining procedures are carried out extravesically (B)

no inadvertent injury to the surrounding structures or tissues. Next, the remaining procedures are performed extravesically while viewing the laparoendoscopic image. The bladder wall is divided along the intravesically made margin line by electric cautery (Photo $3 \mathrm{~A}$ ), and the entire lesion is circumcised and removed en bloc with the perivesical fat and overly- ing peritoneum if necessary. A frozen section of the edge is obtained in preparation to resect more of the wall.

The PLND was performed during the frozen section pathology examination. The template for PLND included the external iliac artery and the internal obturator muscle laterally, the internal iliac artery 

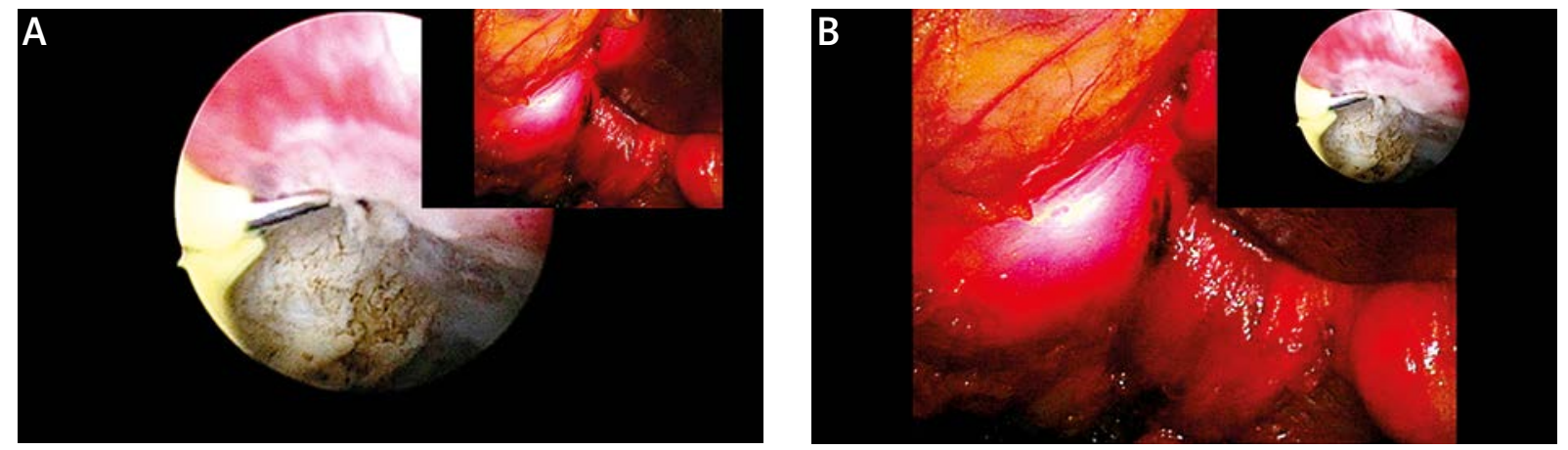

Photo 2. Intravesical approach. The Collins incision is deepened along the proposed resection margin through the serosa until full thickness is achieved. The TUR surgeon carries out this procedure while viewing the cystoscopic image as the main image and the laparoendoscopic image as the second image of the 'Picture in Picture' on the HMD (A), while the other surgeons view the laparoendoscopic image as the main image and the cystoscopic image as the second image to confirm that there is no inadvertent injury to surrounding structures or tissues (B). The TUR site can be easily recognized from outside of the bladder by the transmitted light of cystoscopy passing through the bladder wall
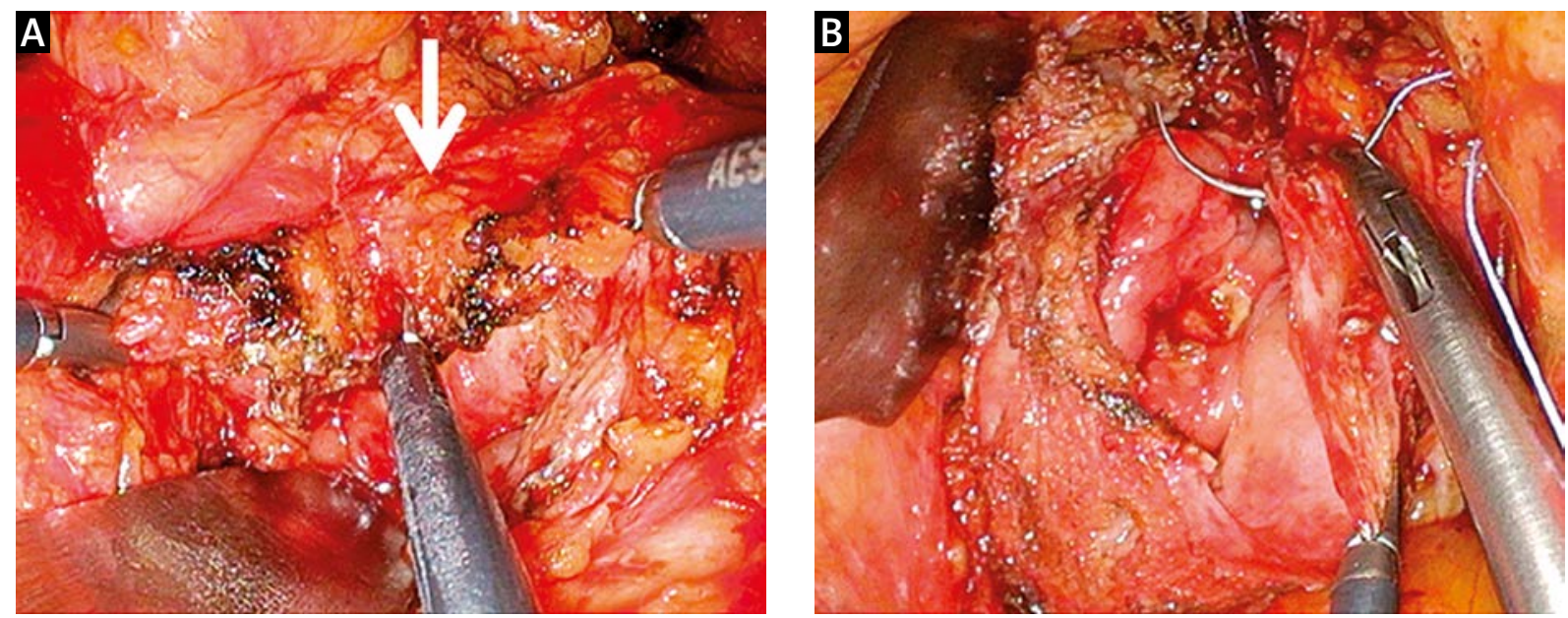

Photo 3. After the intravesical approach, the bladder wall is extravesically divided along the cystoscopically made margin line (arrow) by electric cautery while viewing the laparoendoscopic image (A). The bladder is closed in two layers (B)

medially, the bifurcation of the common iliac artery cranially, and the origin of the epigastric vessels caudally. Finally, the bladder is closed in two layers using a 3-0 Vicryl suture (Photo $3 \mathrm{~B}$ ). The closing site is observed under cystoscopy. Perivesical drains were placed. The abdominal wall was then closed. Postoperatively, the urethral catheter is left in place for 7 to 10 days.

\section{Results}

While carrying out gasless single-port partial cystectomy, the system provided the surgeon with excellent 3D imagery of the operative field. During the surgery, none of the participants experienced any HMD-related adverse effects or reported any discomfort. All five procedures were successfully completed without intraoperative complications (Table I). In 1 patient (\#3), who had a tumor located at the dome, the peritoneum was opened and the entire lesion was removed en bloc with the overlying peritoneum. Frozen section pathology reported atypical urothelial cells in 1 patient (\#2), who had more of the bladder wall resected. The final pathological diagnosis was no cancer cells. Operating times were 215 to 311 (median: 249) min and estimated blood losses were 10 to 125 (median: 50) ml. All patients 
Table I. Clinical features of patients undergoing RoboSurgeon partial cystectomy

\begin{tabular}{|c|c|c|c|c|c|}
\hline Parameter & Patient 1 & Patient 2 & Patient 3 & Patient 4 & Patient 5 \\
\hline Gender & Male & Male & Male & Male & Male \\
\hline Age [years] & 56 & 72 & 74 & 73 & 79 \\
\hline Diagnosis & $\begin{array}{c}\text { High-grade muscle } \\
\text { invasive urothelial } \\
\text { carcinoma } \\
\text { (cT2 pT2 NOMO) }\end{array}$ & $\begin{array}{l}\text { High-grade muscle } \\
\text { invasive urothelial } \\
\text { carcinoma } \\
\text { (cT3b pT2 } \leq \text { NOMO) }\end{array}$ & $\begin{array}{c}\text { High-grade muscle } \\
\text { invasive urothelial } \\
\text { carcinoma } \\
\text { (cT2 pT2 NOMO) }\end{array}$ & $\begin{array}{c}\text { High-grade muscle } \\
\text { invasive urothelial } \\
\text { carcinoma } \\
\text { (cT2 pT2 NOMO) }\end{array}$ & $\begin{array}{l}\text { High-grade muscle } \\
\text { invasive urothelial } \\
\text { carcinoma } \\
\text { (cT2 pT2 NOMO) }\end{array}$ \\
\hline Location & Right lateral wall & Left lateral wall & Dome & Left lateral wall & Left lateral wall \\
\hline Approach & Extraperitoneal & Extraperitoneal & $\begin{array}{l}\text { Extraperitoneal } \\
\text { and } \\
\text { transperitoneal }\end{array}$ & Extraperitoneal & Extraperitoneal \\
\hline $\begin{array}{l}\text { Operative time } \\
\text { [min] }\end{array}$ & 222 & $311^{*}$ & 251 & 215 & 249 \\
\hline $\begin{array}{l}\text { Estimated blood } \\
\text { loss [ml] }\end{array}$ & 70 & 30 & 125 & 10 & 50 \\
\hline $\begin{array}{l}\text { Intraoperative } \\
\text { complications }\end{array}$ & None & None & None & None & None \\
\hline $\begin{array}{l}\text { Postoperative } \\
\text { complications }\end{array}$ & None & None & $\begin{array}{l}\text { Lymphorrhea } \\
\text { requiring drain } \\
\text { insertion }\end{array}$ & None & None \\
\hline Final pathology\# & $\begin{array}{l}\text { No viable cancer } \\
\text { cells in PC and } \\
\text { PLND specimens }\end{array}$ & $\begin{array}{l}\text { No viable cancer } \\
\text { cells in PC and } \\
\text { PLND specimens }\end{array}$ & $\begin{array}{l}\text { No viable cancer } \\
\text { cells in PC and } \\
\text { PLND specimens }\end{array}$ & $\begin{array}{l}\text { No viable cancer } \\
\text { cells in PC and } \\
\text { PLND specimens }\end{array}$ & $\begin{array}{l}\text { No viable cancer } \\
\text { cells in PC and } \\
\text { PLND specimens }\end{array}$ \\
\hline
\end{tabular}

${ }^{\star} A$ frozen section of the edge showed atypical urothelial cells, and more of the bladder wall was resected. The final pathological diagnosis was no cancer cells. \#PC - partial cystectomy, PLND - pelvic lymph node dissection

had the urethral catheter removed within 10 days. Postoperative recovery was uneventful except for 1 patient (\#3) who developed lymphorrhea that required drain reinsertion.

\section{Discussion}

The da Vinci Surgical Robotic System (Intuitive Surgical, Sunnyvale, CA, USA) is increasingly being used to perform urologic surgery. Robotic technology provides additional dimensions to laparoscopy by adding 3D observation, improved ergonomic stabilization of surgical movements, and ease of intracorporeal suturing. This system has several aspects that need to be improved for structural or financial reasons, such as a large imaging console located some distance from the patient, a lack of tactile feedback and extremely high equipment and running costs.

To preserve the benefits and mitigate the defects of the systems, we recently developed an affordable new 3D vision system (RoboSurgeon System), which is a combination of a high-definition 3D organic electroluminescent HMD and a high-definition 3D endoscope. We first applied this system to gasless single-port radical nephrectomy [7]. We subsequently applied it to various urologic procedures, including transurethral resection of the prostate [8]. This is the first study to use this system in gasless laparoendoscopic single-port partial cystectomy. The high-definition 3D endoscope, which enables clear depth perception, is beneficial for complex tasks during the surgery. Using a 3D HMD instead of a standard 3D TV display, surgeons can rely on natural lines of sight, with the head at any desired angle of rotation or inclination, which minimizes fatigue. The surgeon wearing the HMD carries out the surgery while touching and looking at the patient and receiving tactile feedback through the device.

The da Vinci Surgical Robotic System was recently reported to have been applied to partial cystectomy not only in patients with benign lesions, such as bladder pheochromocytoma and leiomyoma, but also in selected patients with bladder cancer $[9,10]$. For example, Allaparthi et al. reported 3 cases of bladder cancer patients who underwent transperi- 
toneal robotic partial cystectomy [9]. The robotic partial cystectomy was completed in all 3 patients without the need for open conversion. However, 1 patient was readmitted and underwent small bowel resection secondary to bowel obstruction. One of the possible advantages of our RoboSurgeon partial cystectomy is that it is typically performed using an extraperitoneal approach, which is unlikely to be associated with bowel complications. Even when the tumor is located at the dome, the opening of the peritoneum is very restricted.

RoboSurgeon gasless single-port partial cystectomy is carried out with a technique that employs both an intravesical and extravesical approach, which we believe has some advantages. First, this prevents overdissection of the bladder. The site of the tumor can be easily recognized from the outside of the bladder by the transmitted light of the cystoscope passing through the bladder wall. Second and most importantly, the margin can accurately be identified under cystoscopy. After the intravesical excision, extravesical excision of the remaining tissue can easily be performed. In our system, each participant can independently rearrange the images comprising the 'Picture in Picture' image depending on the surgical step, enabling the surgery to be performed easily and safely. During the intravesical approach, the TUR surgeon sees the cystoscopic image as the main image, while the other surgeons see the laparoendoscopic image as the main image to confirm that there is no inadvertent injury to surrounding structures or tissues.

In our series, none of the patients had viable cancer cells in either PC or PLND specimens in the final pathology. We do not consider that this means that PC with PLND was not necessary in these patients. As mentioned in the introduction, PC with PLND could contribute to eradication of subclinical residual disease in original MIBC sites and micrometastases in pelvic lymph nodes [2-4]. In our previous series of 46 patients undergoing PC with PLND in our bladder preservation protocol, none developed MIBC recurrence in the preserved bladder or pelvic lymph node recurrence $[2,3]$.

\section{Conclusions}

This study demonstrates the technical feasibility and utility of gasless laparoendoscopic single-port partial cystectomy using our 3D HMD system. A study consisting of a larger number of patients and longer follow-up is needed to validate the utility and establish the oncological outcomes of this technique in patients with bladder cancer.

\section{Conflict of interest}

Dr. Kihara has received research funding from Sony Corporation (Tokyo, Japan), but the sponsor had no control over the interpretation, writing, or publication of this work.

\section{References}

1. Rödel C, Weiss C, Sauer R. Trimodality treatment and selective organ preservation for bladder cancer. J Clin Oncol 2006; 24: 5536-44.

2. Koga F, Kihara K, Yoshida S, et al. Selective bladder-sparing protocol consisting of induction low-dose chemoradiotherapy plus partial cystectomy with pelvic lymph node dissection against muscle-invasive bladder cancer: oncological outcomes of the initial 46 patients. BJU Int 2012; 109: 860-6.

3. Koga F, Kihara K. Selective bladder preservation with curative intent for muscle-invasive bladder cancer: a contemporary review. Int J Urol 2012; 19: 388-401.

4. Koga F, Fujii Y, Masuda $\mathrm{H}$, et al. Pathology-based risk stratification of muscle-invasive bladder cancer patients undergoing cystectomy for persistent disease after induction chemoradiotherapy in bladder-sparing approaches. BJU Int 2012; 110: E203-8.

5. Kihara K, Kageyama Y, Yano M, et al. Portless endoscopic radical nephrectomy via a single minimum incision in 80 patients. Int J Urol 2004; 11: 714-20.

6. Kihara K, Kawakami S, Fujii Y, et al. Gasless single-port access endoscopic surgery in urology: minimum incision endoscopic surgery, MIES. Int J Urol 2009; 16: 791-800.

7. Kihara K, Fujii Y, Masuda $H$, et al. New three-dimensional head-mounted display system, TMDU-S-3D system, for minimally invasive surgery application: procedures for gasless single-port radical nephrectomy. Int J Urol 2012; 19: 886-9.

8. Yoshida S, Kihara K, Takeshita H, Fujii Y. A head-mounted display-based personal integrated-image monitoring system for transurethral resection of the prostate. Videosurgery Miniinv in press.

9. Allaparthi S, Ramanathan R, Balaji KC. Robotic partial cystectomy for bladder cancer: a single-institutional pilot study. J Endourol 2010; 24: 223-7.

10. Tareen BU, Mufarrij PW, Godoy G, Stifelman MD. Robot-assisted laparoscopic partial cystectomy and diverticulectomy: initial experience of four cases. J Endourol 2008; 22: 1497-500.

Received: 27.02.2014, accepted: 7.04.2014. 